\title{
Measurement of dynamic beam-beam effects
}

\author{
T. Ieiri, Y. Funakoshi, T. Kawamoto, M. Masuzawa, and M. Tobiyama \\ KEK, 1-1 Oho, Tsukuba, Ibaraki 305-0801, Japan
}

(Received 22 August 2005; published 9 December 2005)

\begin{abstract}
A series of measurements were carried out to investigate the dynamic beam-beam effects at KEKB. The beam-beam kick and the coherent beam-beam tune shift were measured by comparing the beam parameters between a colliding bunch and a noncolliding one. We found that collisions occur with a horizontal orbit offset to obtain the maximum luminosity, and that this reproducible offset appears to be attributed to an electron cloud effect, which might play an important role in the beam-beam interaction. The horizontal beam size estimated from the beam-beam kick slightly decreased as the beam-beam parameter increased. The horizontal emittance estimated from the beam-beam tune shift was higher than an unperturbed emittance, and agreed with the calculated emittance using a higher-mode tune. The ratio of the square-root betatron function between two locations in the ring was measured as a function of the beam-beam parameter. These experimental results would provide useful information on dynamic beambeam effects.
\end{abstract}

DOI: 10.1103/PhysRevSTAB.8.124401

PACS numbers: 29.20.Dh, 29.27.Bd

\section{INTRODUCTION}

KEKB $[1,2]$ is a multibunch, high-current, electron/ positron collider for $B$-meson physics. The collider consists of two storage rings: the Low Energy Ring (LER) for a 3.5-GeV positron beam and the High Energy Ring (HER) for $8-\mathrm{GeV}$ electrons. Both rings store more than 1300 bunches, where the harmonic number is 5120 with an $\mathrm{rf}$ frequency of $509 \mathrm{MHz}$. The two beams collide at one interaction point (IP) with a horizontal crossing angle of 22 mrad. Unlike conventional single-ring colliders, the beam parameters are different between the two rings, which makes collisions complicated. The maximum luminosity achieved so far is $1.58 \times 10^{34} \mathrm{~cm}^{-2} \mathrm{sec}^{-1}$, which is the best in the world. The main parameters of KEKB are listed in Table I.

The LER has suffered from a blowup in the vertical beam size due to the electron cloud since an early stage of commissioning. Solenoids were installed in all spaces where installation was feasible. As a result, the instability was significantly improved. However, an effect of the electron cloud is still observed in the vertical direction, when a high beam current with a narrow bunch spacing is stored [3]. It is important to investigate the interaction between the electron cloud and beam-beam collisions. On the other hand, a beam-beam interaction changes the emittance and the betatron function due to the dynamic effect, since KEKB is operated at the horizontal betatron tune just above a half integer with a high beam-beam parameter. The beam parameters might vary, depending on the beam intensity and the beam size. It is also important to obtain an experimental verification of the dynamic beam-beam effects from the viewpoint of beam dynamics.

The beam-beam collisions are detected by beamposition monitors installed at both sides of the IP [4]. The monitors detect an orbit change at the IP by measuring the average orbit for all bunches. The orbit of the electron beam at the IP is controlled using dipole magnets near the IP, called iBump magnets. Operational experience shows that the luminosity is very sensitive for the horizontal orbit at the IP, even in the case of a flat beam. When the horizontal strength of the iBump magnets was set below a limit, we observed a sudden decrease in the luminosity, called egure [5], accompanied by an increase of the vertical size of the positron beam. Thus, the horizontal strength of the iBump magnets is limited within a region to keep a stable operation, while monitoring the vertical size of the positron beam.

In order to investigate any dynamic beam-beam effects and mysterious phenomena in collision tuning, the beambeam effects were measured by comparing the beam parameters between collision and noncollision bunches. This type of monitor can easily detect the beam-beam effects, and is not necessary to install near the complicated IP region. The next section describes the beam-beam effects: the beam-beam kick, the beam-beam tune shift and dynamic effects. Section III briefly describes the hardware of the gated monitors and their performance. Section IV presents the results obtained in the horizontal orbit scan at the IP. Section V discusses the source of the egure and the dynamic beam-beam effects.

\section{BEAM-BEAM EFFECTS}

A beam-beam collision induces a nonlinear field at the IP, which can be approximated by a quadrupole at small beam-beam separation. The additional field distorts the optical parameters in the ring. The beat of the betatron function at a phase advance of $\Delta \phi_{s}$ from the IP is given by

$$
\Delta \beta(s)=\frac{\beta(s) \beta^{*}}{2 \sin (2 \pi \nu)} \Delta K^{*} \cos \left(2 \pi \nu-2\left|\Delta \phi_{s}\right|\right),
$$


TABLE I. Machine parameters of KEKB.

\begin{tabular}{lccc}
\hline \hline \multicolumn{1}{c}{ Parameter } & LER & HER & \\
\hline Beam energy, $E$ & 3.5 & 8.0 & $\mathrm{GeV}$ \\
Momentum spread, $\delta_{\epsilon}$ & 7.3 & 6.7 & $\times 10^{-4}$ \\
Horizontal emittance, $\epsilon_{x}$ & 18 & 24 & $\mathrm{~nm}$ \\
Betatron tune, $\nu_{x} / \nu_{y}$ & $45.51 / 43.54$ & $44.51 / 41.58$ & $\mathrm{~cm}$ \\
Beta's at IP, $\beta_{x}^{*} / \beta_{y}^{*}$ & $59 / 0.65$ & $56 / 0.62$ & $\times 10^{-4}$ \\
Momentum compaction, $\alpha$ & 3.4 & 3.4 & $\mathrm{~mm}$ \\
Bunch length, $\sigma_{l}$ & $6.5-8.0$ & $6.5-7.0$ & \\
Synchrotron tune, $\nu_{s}$ & 0.025 & 0.021 & $\times 10^{10}$ \\
Particles per bunch, $N_{b}$ & $2.5-6.2$ & $1.9-5.6$ & $\mathrm{~ns}$ \\
Bunch spacing, $S_{b}$ & $5.9-7.9$ & $5.9-7.9$ & $\mathrm{~m}$ \\
Circumference, $C$ & 3016 & 3016 & $\mathrm{MHz}$ \\
Rf frequency, $f_{r f}$ & 508.886 & 508.886 & \\
Harmonic number, $h$ & 5120 & 5120 & \\
\hline \hline
\end{tabular}

where $\Delta K^{*}$ is the field gradient due to the beam-beam force and $\nu$ is the betatron tune; $\beta(s)$ and $\beta^{*}$ are the betatron functions at a phase advance of $\Delta \phi_{s}$ and at the IP, respectively. The beam-beam collision also changes the emittance of both beams. The variations strongly depend on the betatron tune. Figure 1 shows the calculated optical parameters; the relative betatron function at the IP, and the

(a)

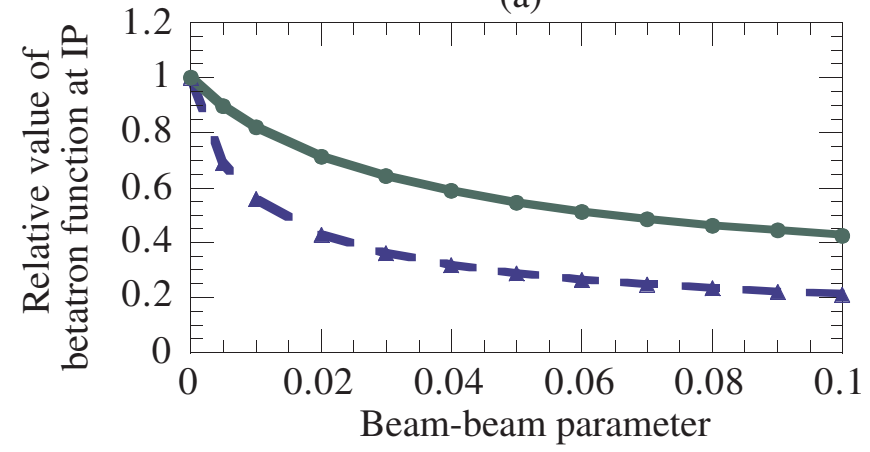

(b)

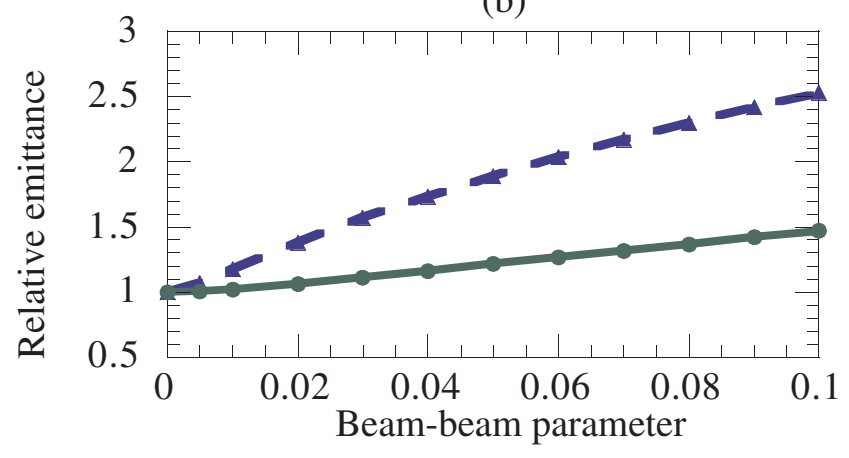

FIG. 1. (Color) Relative value of the betatron function at the IP (a) and relative value of the emittance (b) as a function of the beam-beam parameter. A value of 1.0 corresponds to a nonperturbed value. The green solid line was calculated with a fractional tune of 0.540 , and the blue dashed line with a tune of 0.509 . relative emittance, as a function of the beam-beam parameter with two fractional tunes of 0.509 and 0.540 . The emittance increases, but the betatron function at the IP decreases as the beam-beam parameter increases. Thus, the beam size at the IP slightly decreases, compared with a nonperturbed beam size. These dynamical effects should affect the luminosity performance.

When two beams collide with an offset in the beam orbit at the IP, they are kicked by the space charge of the opposite beam and the orbit is distorted around the ring. A position monitor located at a phase advance of $\Delta \phi_{d}$ from the IP detects an orbit change due to the collision with an offset. The position shift at a detector is given by

$$
\Delta X_{\mathrm{det}}=\frac{\sqrt{\beta_{\mathrm{det}} \beta_{x}^{*}}}{2 \sin (\pi \nu)} \theta_{b b} \cos \left(\pi \nu-\left|\Delta \phi_{d}\right|\right) .
$$

Here, $\beta_{\text {det }}$ is the betatron function at a detector and $\theta_{b b}$ is the beam-beam kick angle. A position shift at a detector is roughly proportional to the beam-beam kick, assuming $\beta_{\mathrm{det}} \beta_{x}^{*}$ is constant. Assuming a rigid Gaussian model, the horizontal beam-beam kick for a bunch is given by [6]

$$
\theta_{b b}\left(\Delta_{x}, \Delta_{y}\right)=\frac{-2 r_{e} N_{b}}{\gamma} \Delta_{x} \int_{0}^{\infty} \frac{\exp \left(-\frac{\Delta_{x}^{2}}{\left(t+2 \Sigma_{x}^{2}\right)}-\frac{\Delta_{y}^{2}}{\left(t+2 \Sigma_{y}^{2}\right)}\right)}{\left(t+2 \Sigma_{x}^{2}\right)^{3 / 2}\left(t+2 \Sigma_{y}^{2}\right)^{1 / 2}} d t .
$$

Here, $\Delta_{x}$ and $\Delta_{y}$ are the horizontal and vertical position offsets at the IP, $N_{b}$ the number of particles in a bunch, $r_{e}$ the classical electron radius, $\gamma$ the relativistic factor, and $\Sigma_{x, y}=\sqrt{\left(\sigma_{x, y}^{+}\right)^{2}+\left(\sigma_{x, y}^{-}\right)^{2}}$ the effective beam size. The superscript \pm denotes positron and electron bunches. Figure 2 shows the horizontal beam-beam kick as a function of the horizontal IP offset, with the effective beam size as a parameter. We can estimate the effective beam size at the IP from the kick curve. Around zero offset, the horizontal beam-beam kick is inversely proportional to square of the effective horizontal beam size for a flat beam. The 


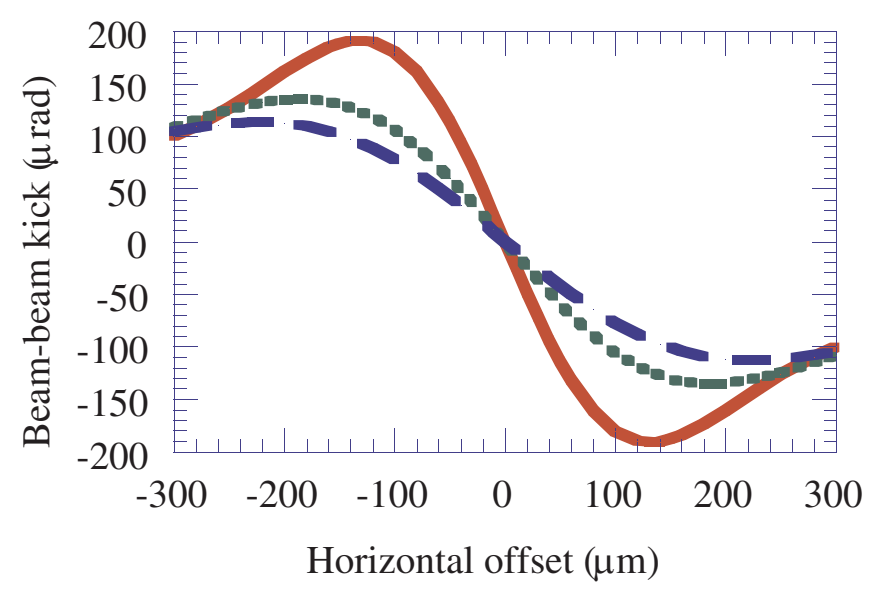

FIG. 2. (Color) Beam-beam kicks as a function of the horizontal offset, assuming an effective horizontal beam size of $100 \mu \mathrm{m}$ (red solid line), $141 \mu \mathrm{m}$ (green dotted line) and $170 \mu \mathrm{m}$ (blue dashed line). The effective vertical size is a constant value of $2.83 \mu \mathrm{m}$ for all cases under the same bunch current.

beam-beam kick has a maximum value at a horizontal offset of $\Delta_{x m} \approx 1.32 \Sigma_{x}$ and its maximum kick is inversely proportional to the beam size under a constant intensity. These features are useful for estimating the beam size. The calculated horizontal sizes without the dynamic effect are $\sigma_{x 0}^{+}=103 \mu \mathrm{m}$ and $\sigma_{x 0}^{-}=116 \mu \mathrm{m}$ at the IP. Therefore, the effective horizontal size is estimated to be $\Sigma_{x 0}=$ $155 \mu \mathrm{m}$.

When the position shift is measured at two locations in a ring for a phase advance of $\Delta \phi_{1}$ and $\Delta \phi_{2}$, the ratio of two position shifts at two locations is given by

$$
\frac{\Delta X_{2}}{\Delta X_{1}}=\sqrt{\frac{\beta_{2}}{\beta_{1}}} \cdot \frac{\cos \left(\pi \nu-\left|\Delta \phi_{2}\right|\right)}{\cos \left(\pi \nu-\left|\Delta \phi_{1}\right|\right)} .
$$

The above equation represents the ratio of the position sensitivity due to a beam-beam kick. The ratio, however, is independent of the beam-beam-kick and gives the ratio of the betatron function including a phase advance at two locations. We might take information on the dynamic betatron function from the ratio of the position shift.

On the other hand, the beam-beam interaction produces a new set of two betatron tunes by mixing two unperturbed tunes. We call these two new modes the higher $(H$-) mode and the lower $(L-)$ mode; both tunes are affected by the beam-beam interaction, unlike the $\Sigma$ - (or zero) and the $\pi$ modes in a single-ring collider. Assuming a rigid Gaussian model, the resultant tunes in the horizontal or the vertical plane are represented by $[7,8]$

$$
\begin{gathered}
\cos \mu_{q H}+\cos \mu_{q L}-\left(\cos \mu_{q 0}^{+}+\cos \mu_{q 0}^{-}\right) \\
=-2 \pi\left(\Xi_{q}^{+} \sin \mu_{q 0}^{+}+\Xi_{q}^{-} \sin \mu_{q 0}^{-}\right),
\end{gathered}
$$

with

$$
\Xi_{q}^{ \pm}=\frac{N_{b}^{\mp} r_{e}}{\gamma^{ \pm}} \frac{\beta_{q}^{* \pm}}{2 \pi \Sigma_{q}\left(\Sigma_{x}+\Sigma_{y}\right)} R_{q}^{ \pm} .
$$

Here, $\mu_{q 0}^{ \pm}$is the betatron phase advance per turn without collision and $R_{q}^{ \pm}$is the reduction factor for the beam-beam parameter due to the crossing angle and the hourglass effect, where the subscript 0 means noncollision, the other subscript $q$ stands for either the $x$ or $y$ plane, and the superscript \pm denotes positron and electron bunches. The unperturbed tune is given by $\nu_{0}^{ \pm}=\mu_{0}^{ \pm} / 2 \pi$. The $H$-mode tune increases as a function of the coherent beam-beam parameter, and the $L$-mode tune stays in between two unperturbed tunes, as shown in Fig. 3. From measuring the coherent beam-beam tune shift, defined by $\Delta \nu_{b b}=$ $\nu_{H}+\nu_{L}-\nu_{0}^{+}-\nu_{0}^{-}$, we could obtain the sum of the coherent beam-beam parameters as

$$
\Xi_{q}^{+}+\Xi_{q}^{-}=\frac{\kappa\left(\nu_{0}^{+}, \nu_{0}^{-}\right)}{Y} \Delta \nu_{b b}
$$

Here, $\kappa\left(\nu_{0}^{+}, \nu_{0}^{-}\right)$is a coefficient determined by unperturbed tunes and $Y$ is the Yokoya factor [9], which is introduced, considering a coherent tune measurement. Assuming that both beam sizes are equal, the relation between the coherent beam-beam parameter and the incoherent beam-beam parameter is given by $\Xi_{q}^{+}+\Xi_{q}^{-}=\overline{\xi_{q}}$, where $\overline{\xi_{q}}$ is an average incoherent beam-beam parameter, $\left(\xi_{q}^{+}+\xi_{q}^{-}\right) / 2$.

The relation between the horizontal emittance and the coherent beam-beam parameter is expressed by

$$
\epsilon_{x}^{+}+\epsilon_{x}^{-}=\frac{r_{e}}{2 \pi}\left(\frac{N_{b}^{+}}{\gamma^{-}}+\frac{N_{b}^{-}}{\gamma^{+}}\right) \frac{1}{\left(\Xi_{x}^{+}+\Xi_{x}^{-}\right)} .
$$

Here, $\beta_{x}^{+}=\beta_{x}^{-}$is assumed, since these parameters have approximately the same value at KEKB, as shown in Table I. We can estimate the sum of the horizontal emittances from the coherent beam-beam tune shift. The calculated emittance without the dynamic effect is

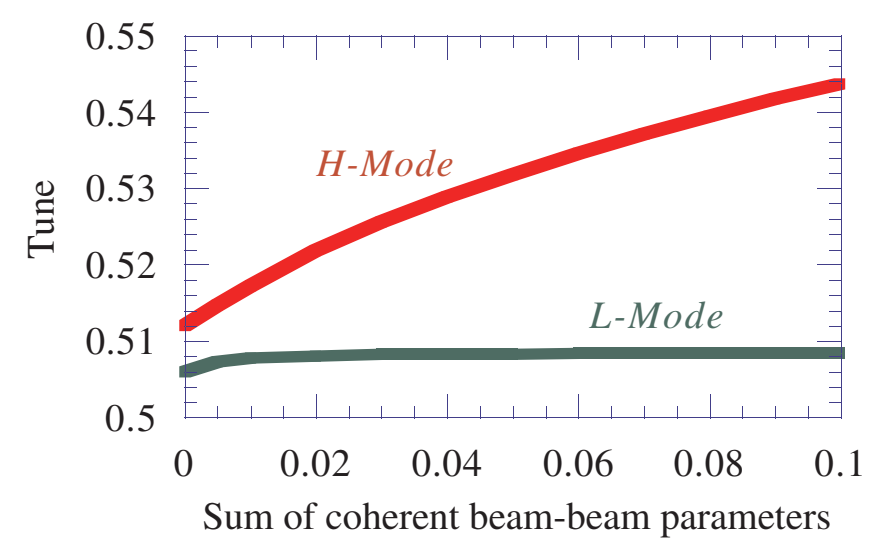

FIG. 3. (Color) $H$-mode and $L$-mode tunes as a function of sum of the coherent beam-beam parameters, where unperturbed fractional tunes are $\nu_{0}^{+}=0.506$ and $\nu_{0}^{-}=0.512$. 
TABLE II. Optical parameters of each monitor, the phase advance from the IP and the betatron function at the monitors without the dynamic beam-beam effects. The position sensitivity, $S_{x \text {-pos }}$ for measuring the horizontal position shift is a relative value, when we set that of LER-2 to be 1.0 as a reference.

\begin{tabular}{lrrrr}
\hline \hline GBPM & LER-1 & LER-2 & HER-1 & HER-2 \\
\hline$\left|\Delta \phi_{x} / 2 \pi\right|$ & 9.34 & 9.77 & 35.35 & 35.12 \\
$\beta_{x}(m)$ & 39.29 & 73.14 & 21.14 & 31.89 \\
$S_{x \text {-pos }}$ & -0.63 & 1.00 & 0.45 & 0.42 \\
$\left|\Delta \phi_{y} / 2 \pi\right|$ & 9.03 & 9.79 & 32.83 & 32.59 \\
$\beta_{y}(m)$ & 3.84 & 5.94 & 7.79 & 3.25 \\
\hline \hline
\end{tabular}

$\epsilon_{x}^{+}=18 \mathrm{nmrad}$ and $\epsilon_{x}^{-}=24 \mathrm{nmrad}$. The sum emittance is $42 \mathrm{nmrad}$.

\section{GATED MONITORS}

KEKB is operated with a single train of bunches followed by an empty gap occupying about $5 \%$ of the circumference. Bunches in a train are usually spaced by 6 or $8 \mathrm{~ns}$. Additional bunches, called pilot bunches, are placed just after the train, at a different location in each ring so that they do not collide with each other. We can easily evaluate the beam-beam effects by comparing the beam parameters of a colliding bunch in a train with those of the noncolliding pilot bunch. However, it would be necessary to consider effects in a single beam, since the beam parameters of different bunches are evaluated. Thus, each parameter with a single beam should be measured under the same bunch structure in advance. This gated measurement has the following features:

(i) We do not install a detector near the complicated IP.

(ii) The position measurement is not affected by the global orbit correction [2].

(iii) Imbalance in gains of the detector is canceled out due to subtraction. (iv) An effect of the wakefield could be removed by considering the measurement in a single beam.

(v) However, the measurement is not simultaneous.

(vi) An error would be enhanced, when the intensity between bunches to be measured is largely unbalanced.

Gated beam-position monitors (GBPM) are installed about $600 \mathrm{~m}$ away from the IP in each ring. Two monitors are prepared for each beam. The monitors detect not only the transverse position, but also the beam phase [10]. Table II gives the optical parameters at the monitors without the dynamic beam-beam effects. One of the monitors, LER-2, located at an optimum phase advance from the IP with a high horizontal betatron function of $73 \mathrm{~m}$, has the highest position sensitivity for detecting the horizontal beam-beam kick. The other monitors have about half the position sensitivity as shown in Table II. The betatron function and the phase advance from the IP, however, might be changed due to the dynamic effects. Based on a rigid Gaussian model, the betatron functions at LER-1 and LER-2 increase as the beam-beam parameter increases. It was found, however, that the product of the horizontal betatron functions at the IP and at LER-2 detector was almost constant, regardless of the beam-beam parameter. Therefore, the horizontal beam-beam kick could be obtained without any influence of the dynamic betatron function.

A GBPM samples a beam signal at a revolution frequency of $100 \mathrm{kHz}$. The beam position and the beam phase are calculated turn by turn, and are stored in a memory. The reproducibility of the averaged values over 32000 turns was obtained under a stable beam. The histograms are shown in Fig. 4. The standard deviation of the positions is about $10 \mu \mathrm{m}$ over 200 data points; note that the measurement includes the stability of the beam itself. The standard deviation of the phase is $0.05^{\circ}$, corresponding to a time resolution of $270 \mathrm{fs}$.

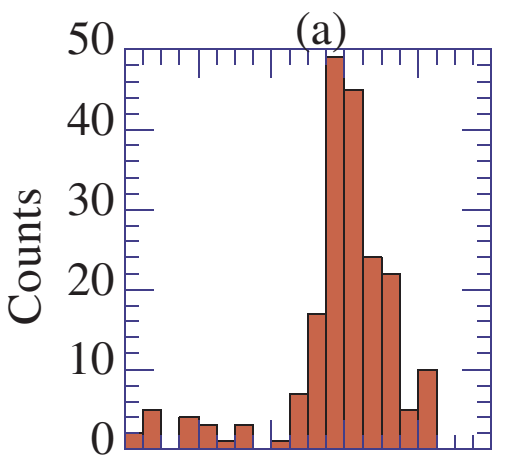

260. 280300320340360 Horizontal position $(\mu \mathrm{m})$

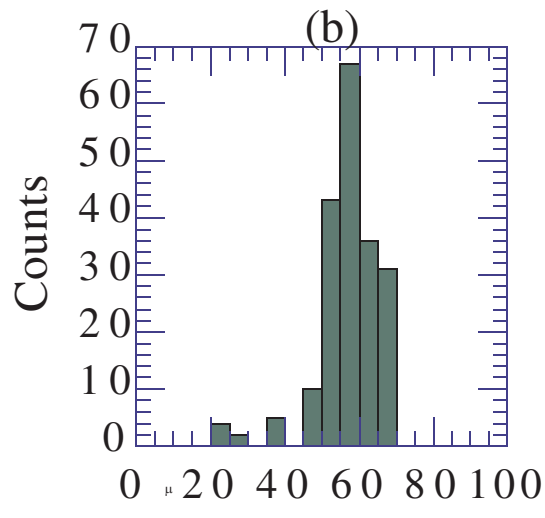

Vertical position $(\mu \mathrm{m})$

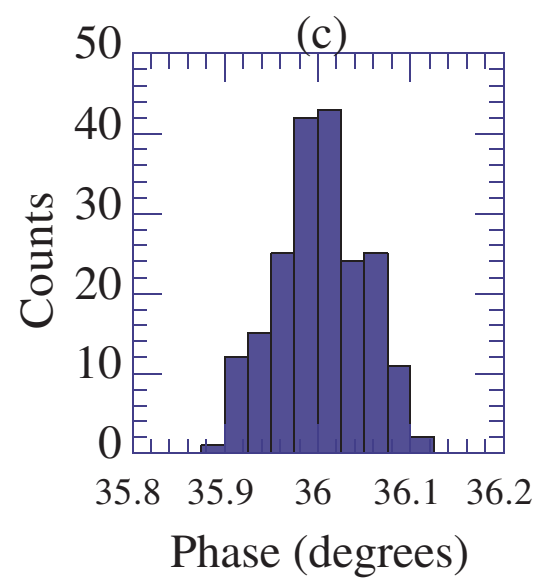

FIG. 4. (Color) Reproducibility of the measured beam positions; (a) the horizontal position, av $=318.6 \mu \mathrm{m}, \mathrm{rms}=15.5 \mu \mathrm{m}$, (b) the vertical position, $\mathrm{av}=59.9 \mu \mathrm{m}, \mathrm{rms}=9.1 \mu \mathrm{m}$, (c) the beam phase, $\mathrm{av}=36.00^{\circ}, \mathrm{rms}=0.05^{\circ}$. 
A gated tune monitor [11] can measure a bunch-bybunch tune by selecting a bunch with a GaAs switch. The tune is measured by a swept frequency method and can be read from the resonant frequency on the spectrum. The tune measurement system is coupled with a transverse feedback loop [12]. The transverse feedback enhances the damping of the betatron oscillation so as to cope with any instabilities, which results in a lowering of the $Q$ value in the tune spectrum. Thus, another gate inserted on the feedback line cuts off the feedback power for a measured bunch. The tune of a pilot bunch is usually measured under normal operation. The tune of a colliding bunch can be measured by changing the timing for the bunch selection.

\section{MEASUREMENT}

\section{A. Measurement during physics runs}

An orbit control at the IP is essential to keep the luminosity at KEKB. The beam-position monitors installed at both sides of the IP detect an orbit offset and a crossing angle at the IP using a beam-beam deflection method [13]. The iBump magnets work to minimize a deviation of the orbit parameters using the beam-position data. The orbit feedback control works well in the vertical direction. In the horizontal direction, however, the orbit feedback with the beam-beam deflection method did not succeed. Instead, the horizontal orbit is controlled using the iBump magnets with mixing two methods empirically developed [5]. One controls the horizontal orbit with a semimanual method so as to maximize the luminosity. The other method uses the vertical size of the positron beam, since the vertical beam size depends on the horizontal offset at the IP, and keeps the vertical size and the luminosity at an optimum level.

A beam-beam effect can be evaluated by a gated-bunch method described in the previous section, where a beam parameter of a colliding bunch is compared with that of a noncolliding one. A GBPM was used to investigate the beam-beam kick. The position shift due to the collision was obtained from subtracting the position of the noncolliding pilot bunch from that of two colliding bunches located next to the pilot bunch. Before subtraction under collisions, a similar subtraction was done to cancel the effect in a single beam under the same bunch pattern. In single-beam mode, the variations in measured beam position along a train were less than $\pm 20 \mu \mathrm{m}$ over the full current range, except for some bunches placed at the leading part of a train and a pilot bunch in the LER. The position shift measured under several beam currents in a single beam was used as an origin of the offset at the IP. Figure 5 shows the position shift detected at LER-2 as a function of the HER total beam current under normal colliding operations. We found that the horizontal position shift changed as a function of the beam current. Considering the source of the position shift, first, a position shift due to beam-induced heating around the ring may be a candidate. However, the effect would be canceled out by

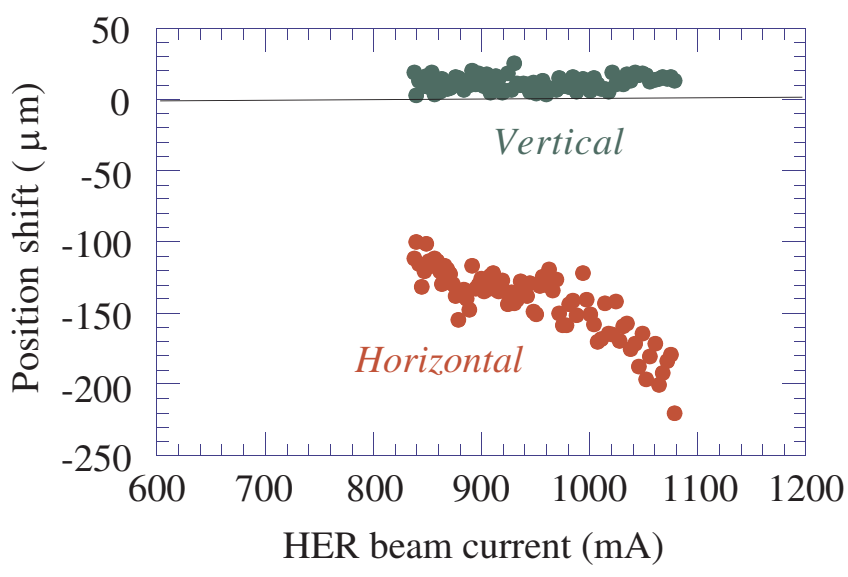

FIG. 5. (Color) Horizontal (red) and vertical (green) position shifts at LER-2 as a function of the HER total beam current, measured in a physics run in December 2003, where a peak luminosity of $1.1 \times 10^{34} \mathrm{~cm}^{-2} \mathrm{sec}^{-1}$ was recorded.

subtracting position data of two bunches in a train. Second, the longitudinal displacement due to the transient beam loading [11] causes the horizontal position shift under a finite crossing angle. A phase difference between the collision and noncollision bunches was measured to be $0.2^{\circ}$ to $0.3^{\circ}$ over the full range of the beam current. A differential phase between both rings stayed in less than $0.1^{\circ}$, which might not have affected the horizontal position shift. Assuming that the current-dependent position shift is caused by a horizontal orbit offset at the IP, a position shift of $-150 \mu \mathrm{m}$ detected at LER-2 is equivalent to an offset of about $40 \mu \mathrm{m}$ at the IP, using calculated betatron functions and an estimated beam-beam parameter of $\xi_{x}=0.07$. On the contrary, the vertical position shift was approximately constant within the measurement resolution. A small offset of about $10 \mu \mathrm{m}$ might be due to an offset of the monitor.

\section{B. Measurement with horizontal scans}

Based on the result obtained during normal physics runs, an experiment to investigate the source of the orbit offset was carried out by scanning the horizontal orbit at the IP. The horizontal orbit of the electron beam was changed using the iBump magnets, while the vertical orbit and the betatron tune of both beams were kept constant. The global orbit correction [2] was off so as to avoid an interaction with the iBump. The horizontal orbit was scanned under three beam conditions as shown in Table III. The condition of scan-1 was a low bunch current of about $1 / 3$ of normal operations. The bunch current of scan- 2 was doubled compared with that at scan-1, while the bunch spacing was the same as that in normal operations. Scan-3 was carried out at a wide bunch spacing.

While scanning, we recorded various parameters related to the collision. The luminosity is an integrated value for all colliding bunches, and the beam size of both beams measured by an interferometer [14] is an averaged value 
TABLE III. Beam conditions used in the horizontal scan, where the bunch spacing and the bunch current are averaged values.

\begin{tabular}{lcccc}
\hline \hline Scan no. & Spacing $S_{b}(\mathrm{~ns})$ & Current $I_{b}^{+} / I_{b}^{-}(\mathrm{mA})$ & Bunches $N_{b}$ & Tune $\nu_{x 0}^{+} / \nu_{x 0}^{-}$ \\
\hline Scan-1 & 7.4 & $0.43 / 0.29$ & 1289 & $0.511 / 0.514$ \\
Scan-2 & 7.4 & $0.78 / 0.67$ & 1289 & $0.511 / 0.512$ \\
Scan-3 & 47.2 & $0.62 / 0.52$ & 203 & $0.510 / 0.508$ \\
\hline \hline
\end{tabular}

over all bunches. The betatron tune and the beam phase as well as the beam position were measured bunch by bunch. The measurement selected bunches placed at the tail part of a bunch train. These results are presented in Figs. 6-8, corresponding to the scanning conditions. We confirmed that the vertical position and the beam phase were almost constant within the measurement resolution, which suggested that the scans were carried out while changing only the horizontal orbit. An effect of the phase shift due to the transient beam loading was negligible, because the horizontal IP scan was carried out at almost fixed beam current.

First, a scan was carried out under the beam condition of scan- 1 as shown in Table III. The scanning was done from a positive offset to a negative side for all cases, because of an asymmetry described later. Figure 6(a) shows the position shift at the monitor (LER-2), and Fig. 6(b) shows the luminosity and the vertical size of the positron beam as a function of the horizontal offset. We notice that the luminosity has the maximum value around the zero offset, however, it slightly reduces in a negative offset, compared with that in a positive offset. The vertical size of the positron beam slightly increased at the negative offset region, however, it suddenly increased by a factor of 2.0 at a negative offset of $-200 \mu \mathrm{m}$. The horizontal sizes of the positron beam and of the electron beam were approximately constant around the zero offset. Figure 6(c) presents the horizontal beam-beam tune shift as a function of the horizontal offset. The maximum tune shift is obtained at the zero offset, which provides the coherent beam-beam tune shift and the sum emittance.

Second, a similar measurement was carried out under the beam condition of scan- 2 in Table III. The results are shown in Figs. 7(a)-7(c). Comparing Fig. 7(a) with Fig. 6(a), a slope around the zero offset in Fig. 7(a) seems to be slightly steeper than that in Fig. 6(a). The positionshift curve in Fig. 7(a) seems to be asymmetrical for the horizontal offset. A clear change can be seen in the luminosity curve. The luminosity had the maximum value not at the zero offset, but at a finite positive offset. When the horizontal offset went through the zero offset, the luminosity suddenly decreased in half. Simultaneously, a severe blowup in the vertical beam size of the positron beam was observed as shown in Fig. 7(b) with a reduced lifetime. The reduction in the luminosity would be related to the size blowup of the positron beam. Moreover, a coherent instability was observed in the vertical tune spectrum of the positron beam. This instability is presumably related to the increase in the vertical beam size and the reduction in the luminosity. We call the phenomena the egure. On the other hand, the electron beam was relatively stable during the scan. Although the horizontal size of the positron beam was approximately constant during the scan, the horizontal size of the electron beam decreased as the offset went through the negative region. Thus the effective horizontal size might slightly change during the scan. The horizontal beam-beam tune-shift curve in Fig. 7(c) has the maximum
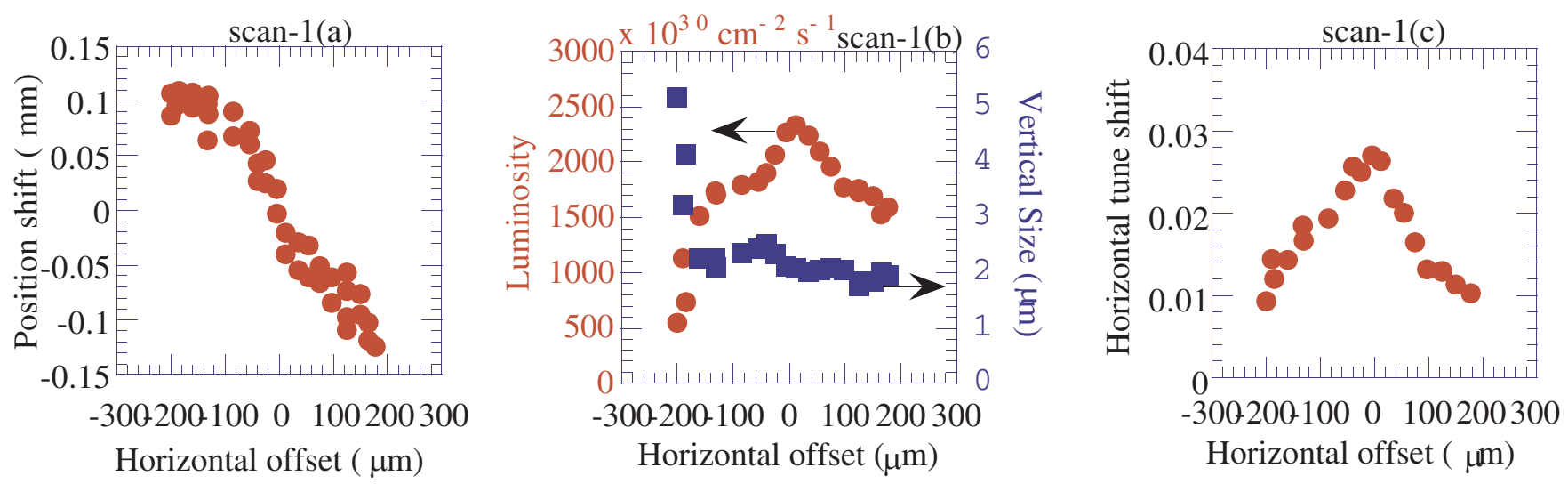

FIG. 6. (Color) Variations of the beam parameters as a function of the horizontal offset with the beam condition of scan-1 (relatively low current). Position shift at LER-2 (a), luminosity (red dots) and vertical beam size of the positron beam at the IP (blue squares) (b), and horizontal beam-beam tune shift (c). 

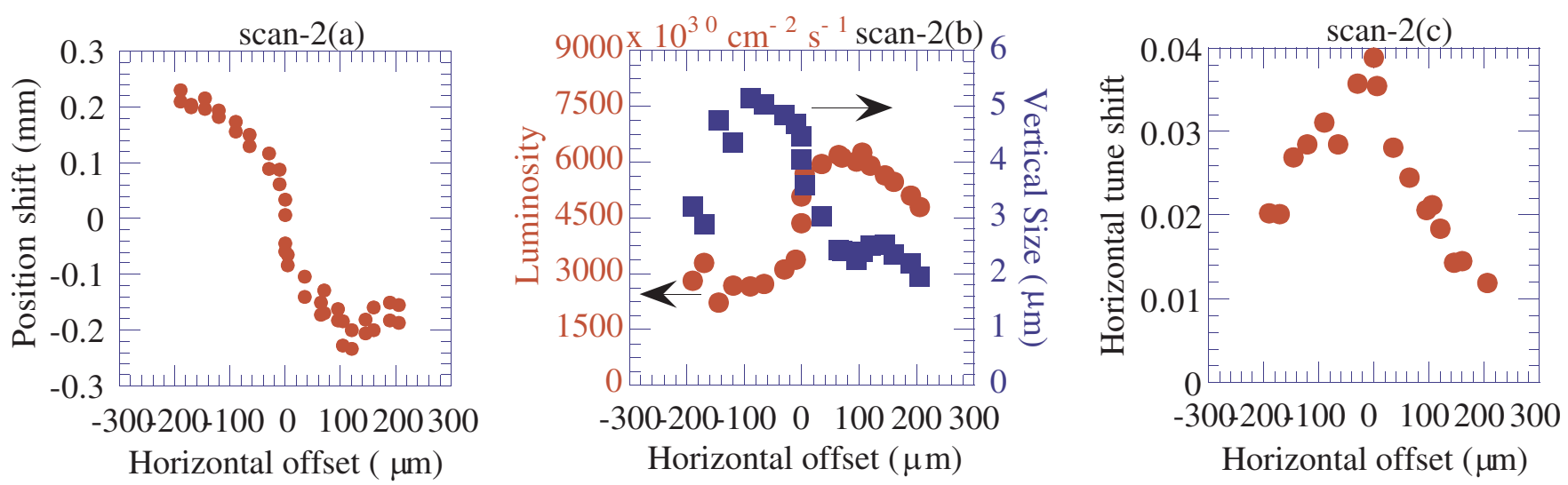

FIG. 7. (Color) Variations of the beam parameters as a function of the horizontal offset with the beam condition of scan-2 (relatively high current). Position shift at LER-2 (a), luminosity (red dots) and vertical beam size of the positron beam at the IP (blue squares) (b), and horizontal beam-beam tune shift (c).

value at the zero offset similarly in the case of scan-1. The tune-shift curve as well as the position shift seems to be asymmetrical for the horizontal offset.

Third, scan-3 was carried out with a wide bunch spacing of 47.2 ns. The results are shown in Figs. 8(a)-8(c). Comparing Fig. 8(b) with Fig. 7(b), a remarkable difference is seen in the luminosity curve, where the luminosity is maximum again at the zero offset and is almost symmetric around the zero offset as shown in Fig. 8(b). At the same time, a blowup of the vertical beam size was not observed when the horizontal offset went through a negative region. The horizontal size of the positron beam was almost constant during the scan. We did not observe a coherent instability in the vertical tune spectrum around the zero offset. It is noted that we tried to start the scan from a negative offset. We observed the egure phenomena in a negative offset under a high beam current of scan-2. Since the positron beam current was largely reduced during a scan due to a reduced lifetime, however, we did not obtain reliable data.

\section{Measurement of the ratio of square root of the betatron function}

The position shift was measured at two locations in the rings, while the horizontal offset at the IP was slightly changed within a region in the positive side to avoid the instability. During the measurement, it was confirmed that the vertical offset was constant and the reduction in the luminosity was less than $6 \%$. The ratio of the position shift, independent of the beam-beam kick, gives square root of the betatron function including the phase advance term at the detectors as given in Eq. (4). Figure 9 shows the ratio measured at the monitors of LER-1/LER-2 as a function of the average beam-beam parameter estimated from tuneshift data. The dynamic betatron function at the monitors was calculated using a rigid Gaussian model and it widely increased by a factor of 3 to 4 with a fractional tune of $\nu_{x 0}=0.510$, when the beam-beam parameter increased to $\xi_{x}=0.08$. The calculated ratios of the betatron function including the phase advance term at the monitors of LER1/LER-2 are also shown in Fig. 9. Although each betatron

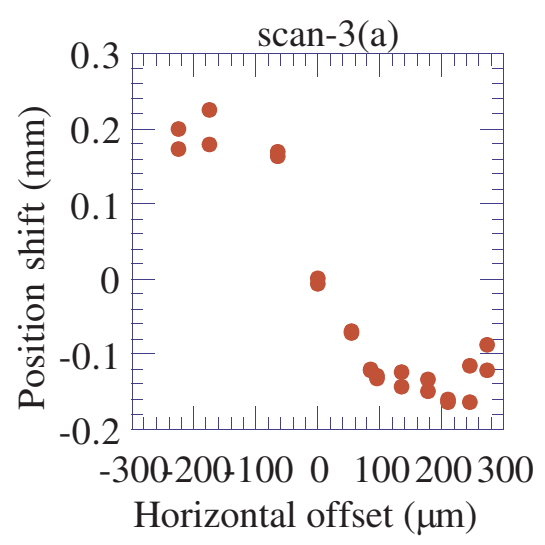

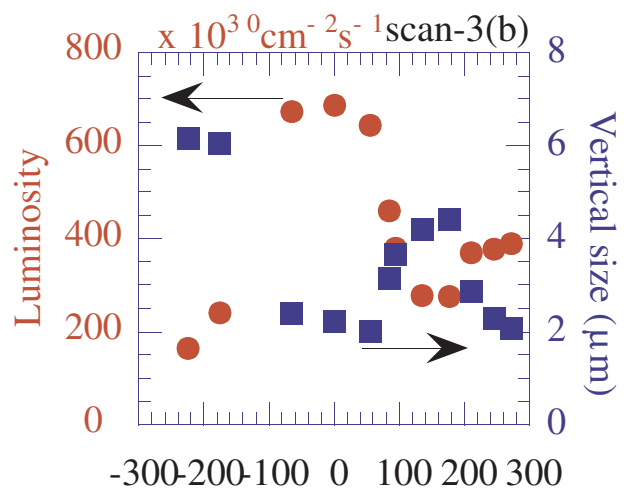

Horizontal offset $(\mu \mathrm{m})$

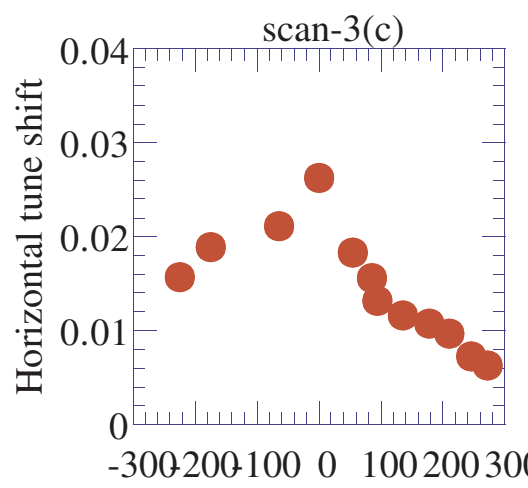

Horizontal offset ( $\mu \mathrm{m})$

FIG. 8. (Color) Variations of the beam parameters as a function of the horizontal offset with the beam condition of scan-3 (a wide bunch spacing of $47.2 \mathrm{~ns}$ ). Position shift at LER-2 (a), luminosity (red dots) and vertical beam size of the positron beam at the IP (blue squares) (b), and horizontal beam-beam tune shift (c). 


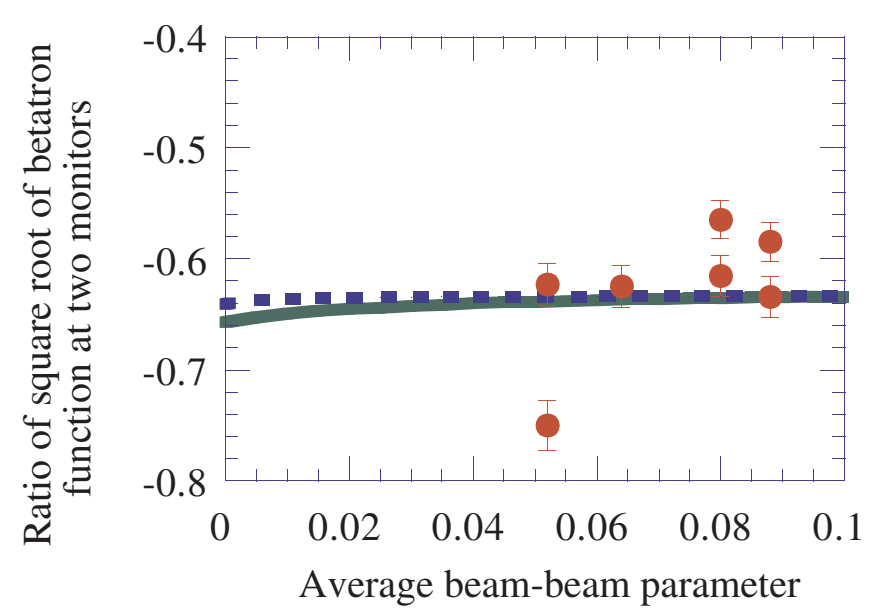

FIG. 9. (Color) Ratio of a square root of the betatron function between two monitors, LER-1/LER-2, as a function of the average beam-beam parameter. The green solid line is the calculated ratio with a fractional tune of 0.540 , and the blue dashed line with a tune of 0.510 , and the red dots with error bars are measured ones.

function widely changed, the ratio was approximately constant. The measured ratio at LER-1/LER-2 approximately agrees with the calculated value.

\section{DISCUSSION}

We observed collisions that occurred with a horizontal offset to obtain the maximum luminosity under normal operations. A position shift of $-150 \mu \mathrm{m}$ at the detector, LER-2, corresponds to an offset of $40 \mu \mathrm{m}$ at the IP, assuming that the horizontal beam-beam parameter is $\xi_{x}=0.07$. Moreover, we found that the luminosity curve was asymmetrical around zero offset in the horizontal scan at a high beam current. However, the asymmetry was not significantly noticed around the zero offset when the beam current was low or the bunch spacing was expanded from 7.4 to $47.2 \mathrm{~ns}$. Figure 10 illustrates our interpretation of why the luminosity is asymmetric for a horizontal scan under normal operations. The beams collide with a horizontal crossing angle of $22 \mathrm{mrad}$ at KEKB. Because of the finite crossing angle, the longitudinal colliding position shifts, depending on the horizontal offset. On the other hand, we understand that a positron bunch experiences a short-range wakefield due to an electron cloud produced by previous bunches in the case of a short bunch spacing [15]. Thus, the tail part of a positron bunch would suffer a more severe wakefield than the head part. In the case of Fig. 10(a), an electron bunch orbits outside a positron bunch at the IP, the head part of a positron bunch mainly collides with the tail of an electron bunch. This is the case of a positive offset, where both beams were experimentally stable. The situation is reverse in Fig. 10(c), in the case of a negative offset. The head part of an electron bunch mainly collides with the tail of a positron bunch where a severe wakefield

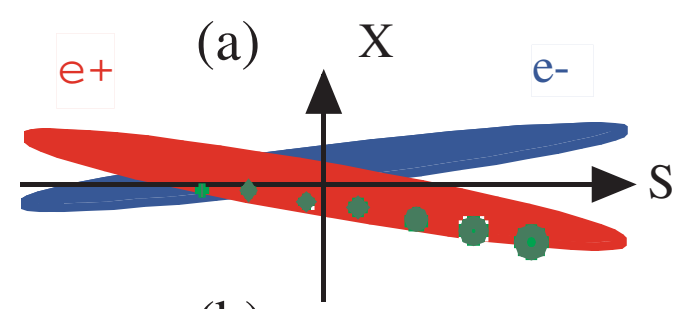

(b)
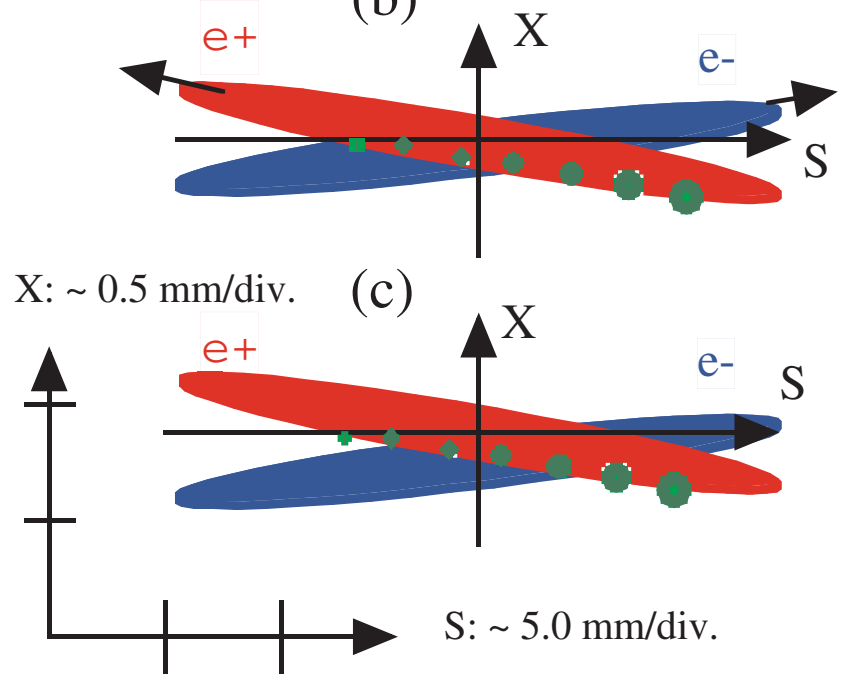

FIG. 10. (Color) Schematic top view at the IP with a horizontal crossing angle. The axis $\mathrm{S}$ indicates the longitudinal direction and $\mathrm{X}$ the horizontal one. The scale factor for both directions is about $1 / 10$. Each figure indicates that the collision occurs with a positive horizontal offset (a), around zero offset (b) and a negative one (c). The red indicates a positron bunch and the blue an electron bunch. The green circles image the wakefield due to the electron cloud, where the size corresponds to the strength of the wakefield.

would exist. In this case, the electron beam would directly stimulate the electron cloud and/or a wakefield in the tail part of a positron bunch, and the whole positron bunch would become unstable via head-tail motion. This situation was experimentally observed as seen in a negative offset region of Fig. 7(b), where the vertical beam size of the positron beam increased and became unstable with a reduced lifetime. The luminosity therefore decreased. When the bunch spacing is wide enough, however, an effect of the electron cloud might weaken, before following bunches arrive. As shown in Fig. 8(b), the luminosity and the vertical size of the positron beam were symmetrical around the zero offset. We did not observe any instability in the positron beam at that time. It is noted here that the effect of parasitic collision is negligible due to the finite crossing angle. Therefore, we could imagine that an effect of the electron cloud under a finite crossing angle could play a rule in producing a vertical instability of the positron beam and an asymmetric curve in the luminosity tuning. On the other hand, a strong-strong beam-beam simulation [16] did not indicate such an asymmetry of the vertical size of the 
positron beam in the horizontal scan. Note that the simulation did not include the effects of the electron cloud and a wakefield due to the ring impedance. A beam-beam simulation including these effects should be required.

To estimate the beam-beam kick from the position shift, the dynamic effect of the betatron function should be considered. Since the production of the perturbed horizontal betatron function including the phase term, $\sqrt{\beta_{x}^{*} \bar{\beta}_{\mathrm{det}}} \cos \left(\pi \nu-\left|\Delta \phi_{d}\right|\right)$ remains approximately constant at $6.4 \mathrm{~m}$ at LER-2, we can easily obtain the beambeam kick from the position shift, regardless of the beambeam parameter. Figure 11 shows the beam-beam kick measured with two different beam currents. Calculated values are also shown based on a rigid Gaussian model, assuming both beam sizes are equal. A calculated curve agrees with the measurement under a low current as shown in Fig. 11(a). However, we cannot fit all values measured into calculated values under a high current as shown in Fig. 11(b), because of an asymmetric beam-beam kick curve. Thus a fitting was carried out only around the zero offset. A fitted curve around the zero offset shows an effective beam size of $155 \pm 15 \mu \mathrm{m}$ in Fig. 11(a) with a
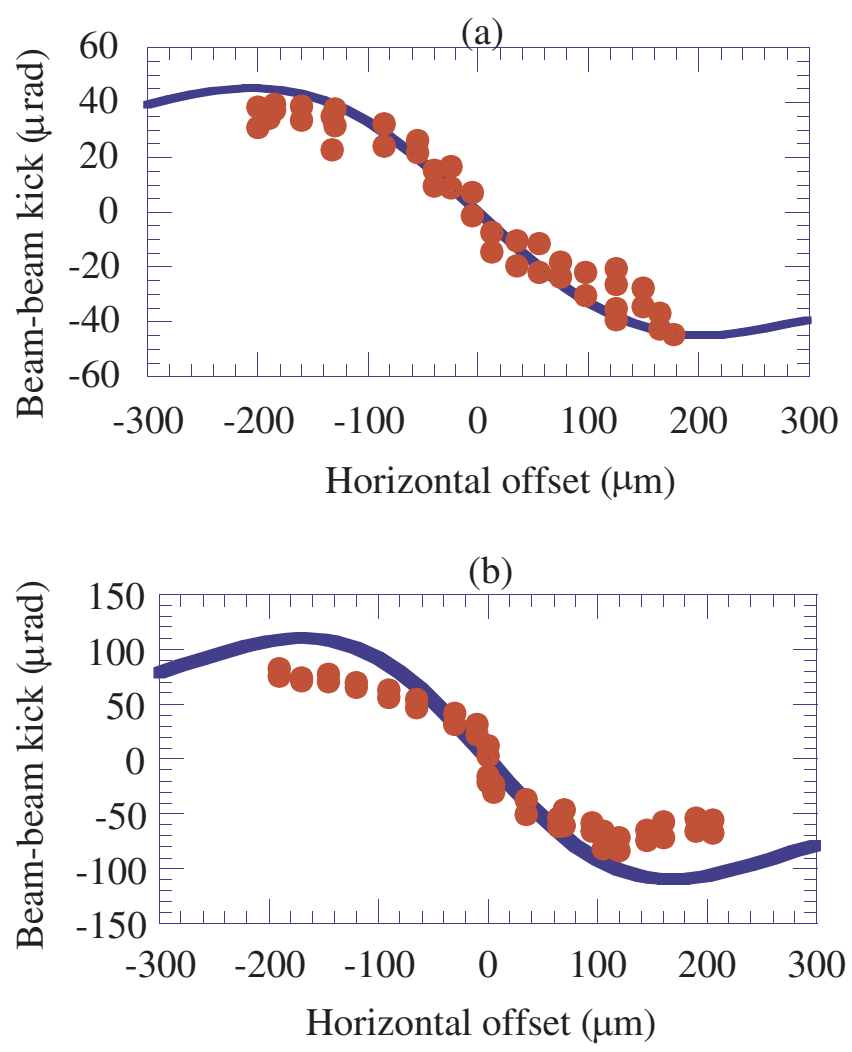

FIG. 11. (Color) The dots are the measured beam-beam kick as a function of the horizontal offset, under the beam condition of scan-1 (a) and scan-2 (b) in Table III. The solid line is the calculated beam-beam kick, assuming each horizontal beam size is equal with $\sigma_{x}^{ \pm}=110 \mu \mathrm{m}$ (a) and $\sigma_{x}^{ \pm}=90 \mu \mathrm{m}$ (b) and the vertical size is $\sigma_{y}^{ \pm}=2 \mu \mathrm{m}$ in both cases. low current and $127 \pm 15 \mu \mathrm{m}$ in Fig. 11(b) with a high current, respectively. Figure 12 shows the fitted effective beam size around the zero offset together with calculated sizes at the IP using two kinds of the tune as a function of the average beam-beam parameter estimated from the beam-beam tune shift. The horizontal beam size at the IP is slightly reduced as the beam-beam parameter increases, although error bars are large. The fitted effective beam size is smaller than that without the dynamic effect at a high beam-beam parameter, where the unperturbed effective size is $\Sigma_{x 0}=155 \mu \mathrm{m}$. This result is consistent with a prediction by the dynamic effect. It is noted, however, that the measured beam-beam kick under a high current suggests an asymmetric beam profile that deviates from a Gaussian distribution. When a bunch placed in the leading part of a train was measured, the asymmetry in the beambeam kick seemed to be weaker than that in a bunch at the tail part. It is required that a realistic beam profile should be considered. On the other hand, the horizontal and the vertical sizes of both beams were measured using the interferometer [14]. The effective horizontal size was not constant during the scan- 2 under a high beam current. The issues on the asymmetric profile and a change of the beam size during the scan remain for future study.

The beam-beam effects change the emittance. The dynamic emittance can be estimated from the coherent beambeam tune shift as given in Eq. (8). Figure 13 shows the sum of the emittances estimated from the tune-shift measurement as a function of the normalized intensity $\left(N_{b}^{+} / \gamma^{-}+N_{b}^{-} / \gamma^{+}\right)$, where $Y=1.31$ is used to obtain the coherent beam-beam parameter. The estimated emittance slightly increases as a function of the normalized intensity. On the other hand, the dynamic emittance can be approximately calculated using the tune [8]. It was confirmed that the approximate dynamic emittance agreed

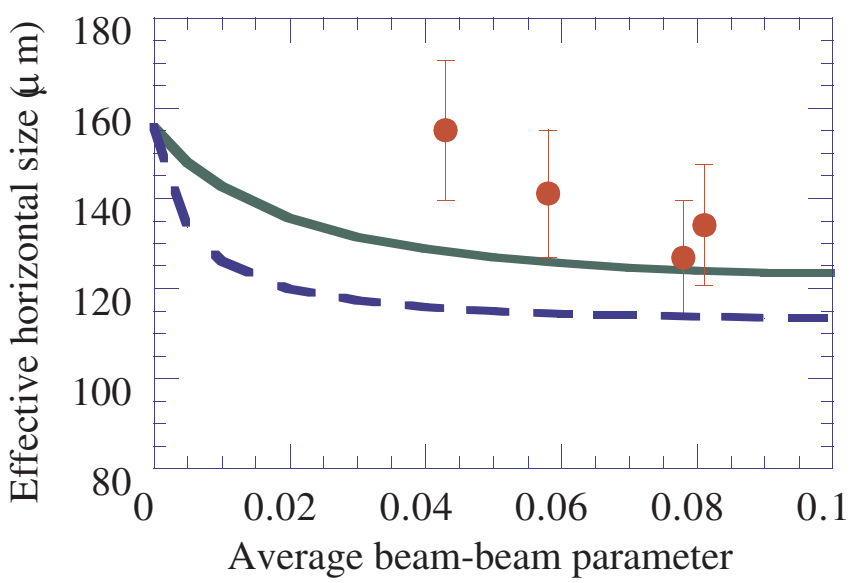

FIG. 12. (Color) The dots with an error bar are the estimated effective horizontal beam size as a function of the average beambeam parameter. The green solid line is the calculated size with a fractional tune of 0.540 and the blue dashed line with a tune of 0.510 . 


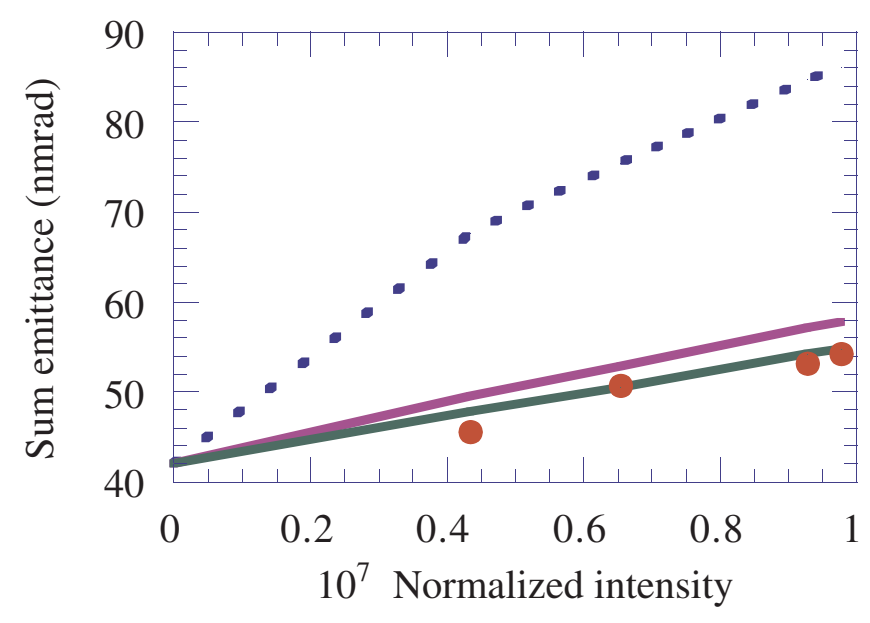

FIG. 13. (Color) Sum of the emittances estimated from the beam-beam tune shift (red dots) and calculated sum emittance with a horizontal tune of 0.512 (blue dashed line), of 0.540 (purple solid line) and of 0.550 (green solid line) as a function of the normalized intensity.

with a value calculated including the whole lattice functions of the rings with less than $10 \%$. Figure 13 also shows the approximate sum emittance as a parameter of the tune. Three values of the tune are used; one is a tune near to the observed lower $(L-)$ mode tune and the others are tunes near to the higher $(\mathrm{H}-)$ mode tune. As the betatron tune approaches a half integer, the dynamic emittance is remarkably enhanced as shown in Fig. 13. The calculated sum emittance without the dynamic effect is $42 \mathrm{nmrad}$. We find that the sum emittance estimated from the tune-shift measurement agrees with the emittance calculated using the $H$-mode tune, not the $L$-mode tune. To confirm this conclusion, a more direct measurement of the individual horizontal emittance would be desirable.

\section{SUMMARY}

The beam-beam effects were investigated with a gatedbunch method at KEKB. We obtained the following experimental results:

(i) Collisions occur with a horizontal orbit offset to avoid the egure phenomena, where the luminosity suddenly decreases in a negative offset, together with a blowup of the vertical beam size of the positron beam.

(ii) We believe that the egure phenomena are caused by a combination of the beam-beam and electron cloud effects under a finite crossing angle.

(iii) The horizontal beam size estimated from the beam-beam kick decreased as the beam-beam parameter increased, which is consistent with the expectation.

(iv) We could estimate an asymmetric horizontal profile that deviates from a Gaussian distribution at a high beam current. (v) The sum of the horizontal emittances estimated from the beam-beam tune shift slightly increased as the beam-beam parameter increased, and agreed with a calculation using the higher $(\mathrm{H}$-) mode tune.

(vi) The dynamic beam-beam effect could be evaluated with the higher $(H-)$ mode tune, not the lower $(L-)$ mode tune.

(vii) The measured ratio of the dynamic betatron function approximately agreed with calculation at two locations of the LER.

\section{ACKNOWLEDGMENTS}

The authors would like to thank Professor K. Oide for his support and fruitful comments. They also thank the members of the KEKB commissioning group for their help. This work was partially supported by a Grant-inAid for Scientific Research (16540271) from Japan Society for the Promotion of Science and Technology.

[1] KEKB Design Report, KEK Report No. 95-7, 1995.

[2] K. Akai, N. Akasaka, A. Enomoto, J. Flanagan, H. Fukuma, Y. Funakoshi, K. Furukawa, T. Furuya, J. Haba, S. Hiramatsu, K. Hosoyama, T. Ieiri, N. Iida, H. Ikeda, S. Kamada, T. Kamitani, S. Kato, M. Kikuchi, E. Kikutani, H. Koiso, S. Kurokawa, M. Masuzawa, T. Matsumoto, T. Mimashi, T. Mitsuhashi, T. Nakamura, Y. Ogawa, K. Ohmi, Y. Ohnishi, S. Ohsawa, N. Ohuchi, K. Oide, E. Perevedentsev, K. Satoh, M. Suetake, Y. Suetsugu, T. Suwada, M. Tawada, M. Tejima, M. Tobiyama, S. Uno, Y. Wu, N. Yamamoto, M. Yoshida, M. Yoshioka, S. Yoshimoto, and F. Zimmermann, Nucl. Instrum. Methods Phys. Res., Sect. A 499, 191 (2003).

[3] H. Fukuma, in ECLOUD'04: Proceedings of the 31st ICFA Advanced Beam Dynamics Workshop on ElectronCloud Effects, Napa, CA, 2004, http://icfa-ecloud04.web.cern.ch/icfa- ecloud04/.

[4] M. Arinaga, J. Flanagan, S. Hiramatsu, T. Ieiri, H. Ikeda, H. Ishii, E. Kikutani, T. Mimashi, T. Mitsuhashi, H. Mizumo, K. Mori, M. Tejima, and M. Tobiyama, Nucl. Instrum. Methods Phys. Res., Sect. A 499, 100 (2003).

[5] M. Tanaka et al., in Proceedings of the 14th Symposium on Accelerator Science and Technology (KEK, Tsukuba, 2003), p. 422.

[6] K. Hirata, Nucl. Instrum. Methods Phys. Res., Sect. A 269, 7 (1988).

[7] K. Hirata and E. Keil, Nucl. Instrum. Methods Phys. Res., Sect. A 292, 156 (1990).

[8] Handbook of Accelerator Physics and Engineering, edited by A.W. Chao and M. Tigner (World Scientific, Singapore, 1998), p. 135.

[9] K. Yokoya and H. Koiso, Part. Accel. 27, 181 (1990).

[10] T. Ieiri and T. Kawamoto, Nucl. Instrum. Methods Phys. Res., Sect. A 440, 330 (2000). 
[11] T. Ieiri, K. Akai, H. Fukuma, T. Kawamoto, E. Kikutani, and M. Tobiyama, Phys. Rev. ST Accel. Beams 5, 094402 (2002).

[12] M. Tobiyama and E. Kikutani, Phys. Rev. ST Accel. Beams 3, 012801 (2000).

[13] Y. Funakoshi (to be published).
[14] T. Mitsuhashi, J. Flanagan, and S. Hiramatsu, in $K E K$ Proceedings 99-24, $e^{+} e^{-}$Factories99, 2000 (KEK, Tsukuba, 2000), p. 134.

[15] K. Ohmi, F. Zimmermann, and E. Perevedentsev, Phys. Rev. E 65, 016502 (2002).

[16] M. Tawada (private communication). 